\title{
Prescrição Off-Label no Brasil e nos EUA: aspectos legais e paradoxos
}

\author{
Off-label prescriptions in Brazil and in the US: \\ legal aspects and paradoxes
}

Patricia Fernandes da Silva Nobre ${ }^{1}$

${ }^{1}$ Instituto Nacional de Controle de Qualidade em Saúde, Fundação Oswaldo Cruz. Av Brasil 4365,

Manguinhos. 21.040-900

Rio de Janeiro RJ.

patricia.silva@incqs.fiocruz.br

\begin{abstract}
The scope of this study is to review the legal aspects of off-label prescription in Brazil compared to the US regulatory environment, to verify whether or not it derives from the structure of the current administrative regulatory process. It involved a bibliographic study in English and Portuguese in public databases of legislation, articles, books and dissertations using the key words 'offlabel,' 'health law,' 'health regulation,' with respective translations into English. In Brasil and the US, health surveillance institutions have no jurisdiction on medical practices and cannot restrict off-label prescriptions beyond educational campaigns. Indications of use are a recommendation of minimum safety and efficacy for product commercialization, but are not a restrictive factor in practice, which does not exonerate physicians from civil liability lawsuits. Public measures to restrict off-label prescriptions are more common in the control of the incentives and dissemination of information to the medical community. Off-label prescription is a risk-benefit assessment made by the clinician and the pros and cons are described in the literature. Brazilian legislation and its stance on the subject still lack greater investigation and subsequent measures.
\end{abstract}

Key words Health surveillance, Off-label prescription, Health law, Health regulation
Resumo Analisar as características legais da prescrição off-label no Brasil comparando com a realidade regulatória norte-americana de forma a verificar se esta é ou não decorrência da estrutura do processo normativo como se dá na atualidade. Levantamento bibliográfico em português e inglês em bases de dados públicas sobre legislação, artigos, teses e livros usando as chaves de busca off-label, direito sanitário, regulação sanitária, com as respectivas traduções para o inglês. No Brasil e nos EUA os órgãos de vigilância sanitária de produtos não têm competência legal para intervir na prática médica em si e não podem restringir a prescrição off-label para além das ações educativas. Dentro do processo regulatório, a indicação de uso é um desfecho a partir do qual se determina segurança e eficácia minimas para comercialização, mas não é fator restritivo prático, o que não exime os profissionais de ações legais por responsabilidade civil. As ações públicas para restringir o uso offlabel são mais frequentes no controle à divulgação e incentivo desse tipo de uso à comunidade médica. O uso off-label é uma conta de risco-benefício feito pelo clínico assistente e que tem prós e contras descritos na literatura. A legislação brasileira e sua postura ainda carecem de maior observação e posterior desenvolvimento.

Palavras-chave Vigilância Sanitária, Prescrição off-label, Direito Sanitário, Regulação Sanitária 


\section{Introdução}

O que significa legalmente e sob o ponto de vista do processo de registro de produtos a indicação de uso? E o que significa a prescrição de produtos para usos diferentes dos utilizados no processo de registro na prática clínica? A compreensão do papel das indicações de uso no processo regulatório permite entender os aspectos de legalidade pertinentes às prescrições médicas de medicamentos e produtos para a saúde na prática clínica.

Podemos definir mais precisamente a prescrição off-label ou não licenciada como a prescrição de medicamentos ou de produtos correlatos para indicações, usos e finalidades distintos daqueles constantes na bula aprovada pela autoridade sanitária competente ${ }^{1-21}$. Conforme diversos autores, a prescrição off-label é frequente em diversas especialidades médicas ${ }^{3,4}$. Pode-se diferenciar o uso off-label do uso experimental ou em pesquisa, sendo esta o uso de produtos novos ainda em fase de estudos, ou mesmo livremente comercializados dentro do contexto do protocolo de uma pesquisa ${ }^{6}$.

Compreendendo a prática de prescrição de produtos e medicamentos off-label como um fenômeno global, esta revisão usa a comparação entre as realidades norte-americana e brasileira para entender como esta prática pode derivar do processo regulatório. Tal questionamento impõe uma relativização nos debates sobre o uso das indicações de uso como um dos critérios para adoção de medicamentos em políticas públicas, ressarcimento do custo de medicamentos e produtos para saúde por seguros, dentre outros, contribuindo para novas formulações críticas e éticas ${ }^{3}$.

\section{Metodologia}

A revisão se detém sobre a legislação pertinente no Brasil e nos Estados Unidos, bem como na literatura acerca das prescrições off-label, em especial a publicada após 1997, ano da edição do Food and Drug Modernization Act norte Americano, que definiu normas para controle de promoção para prescritores nos $\mathrm{EUA}^{8}$; usa a realidade norte-americana como comparação, em virtude de Brasil e Estados unidos praticarem modelos agenciais para controle de produtos para a saúde e a hipótese de que a autoridade reguladora brasileira, segundo a literatura, ter sido inspirada no modelo estadunidense $\mathrm{e}^{9-11}$.

Usando como chave de busca os descritores off-label, off-label prescription e prescrição de medicamentos, foi feito um levantamento da legislação editada no Brasil e nos EUA desde a segunda metade dos anos 90, bem como artigos, livros, teses e dissertações disponíveis em bases de dados de acesso público.

\section{Resultados}

A Anvisa segue o procedimento de controle de drogas norte americano em suas linhas gerais; a autoridade sanitária estadunidense submete os medicamentos por um longo processo de aprovação para liberação para o mercado, no qual são testados quanto à segurança e eficiência. $\mathrm{A}$ avaliação dos produtos é feita com relação à segurança e a finalidades terapêuticas, que podem ser a cura, a melhora de determinadas doenças ou agravos à saúde, ou mesmo serem produtos essencialmente equivalentes aos existentes no mercado, mas que ofereçam diferenciais na dinâmica do tratamento, benefícios a uma população específica, menores efeitos colaterais etc. A segurança e a eficácia são estabelecidos mediante estudos que culminam em ensaios clínicos extensos, com milhares de participantes ${ }^{12}$. Este sistema se inicia em estudos pré-clínicos em animais e prossegue com o pedido de investigação de novas drogas, em estudos de fase 1 , onde o objetivo principal é determinar como a droga é metabolizada e excretada, identificando efeitos colaterais significativos e toxicidade. Uma vez aprovada em fase 1, é possível avançar para a fase 2, cujo objetivo é obter dados primários sobre a eficiência para uma dada condição ou finalidade. Estudos de fase 2, aprofundam dados de segurança e são executados contra placebo utilizando grupos de algumas dúzias até algumas centenas de participantes. Aprovado em fase 2, o medicamento é aceito para submissão a estudos de fase 3, que reúnem maiores informações em diferentes populações sobre segurança, eficiência e dosagens, atingindo até milhares de participantes. Existem estudos de fase 4, que são conduzidos pós-comercialização e que exploram novas populações, novas dosagens, novos usos, e outras situações de uso de produtos que podem ser incorporadas à bula do produto 8,9 . Os estudos são conduzidos considerando uma indicação de uso para o produto, que opera como um modo de se determinar para qual enfermidade ou condição o produto será direcionado, e assim determina-se a população de estudo, resultados (desfecho) esperados, etc. ${ }^{13}$. A indicação de uso é essencial ao processo regulatório como parte do 
desenvolvimento dos estudos de segurança e eficácia dos produtos auxiliando a resposta das perguntas básicas: o produto é seguro? O produto funciona para alguma coisa (não é inócuo) ${ }^{14}$

Estudos de fase 4 não são comumente realizados, já que são facultativos à empresa responsável pelo pedido de autorização de comercialização dos produtos (registro) ${ }^{14}$. Em termos de garantia, segurança e eficiência de medicamentos é o sistema até agora tido como mais confiável; no entanto tem desvantagens por ser lento e caro, e envolver decisões regulatórias que envolvem custos Tipo I e II.

Os riscos da aprovação de medicamentos estão associados à incerteza sobre a segurança e a eficácia dos produtos diante das condições de uso não avaliadas durante o processo de aprovação pela autoridade regulatória e a possibilidade de efeitos adversos não previstos. Quem quer que decida tem de enfrentar o ônus de não aprovar medicamentos que poderiam ter salvado vidas (um custo Tipo I) ou de prescrever medicamentos potencialmente inseguros (um custo Tipo II). A questão reside em saber quais são os custos Tipo II que podem gerar um nexo de causalidade facilmente identificável entre uso e óbito ou agravo (o ato gera o agravo) e expõe aquele que decide ao escrutínio público e à devida responsabilização legal e administrativa. No caso de custos Tipo I, a relação de causalidade é mais diluída (a recusa em tomar determinada decisão poderia ser o gerador do agravo), entrando no reino da possibilidade e quem decide tende a se escudar em práticas mais conservadoras ${ }^{1,2,5,15-17}$.

A partir da emergência de doenças como o HIV/AIDS e da organização de grupos de direitos dos pacientes (patient advocacy groups), riscos tipo I estão mais expostos ao público e a sociedade mais alerta sobre os riscos como uma escolha relativa àqueles que sofrem os danos da doença e há mais pressão para liberação de novos medicamentos, ou mesmo para usos off-label, havendo natural pressão sobre os membros do legislativo - no caso norte-americano o resultado foi a publicação de novas leis que tornaram a aprovação de medicamentos mais rápida e oferecendo incentivos a pesquisa de drogas órfãs e para pediatria ${ }^{17}$.

Os benefícios mencionados na literatura do uso off-label e que costumam fundamentar seu uso por médicos são:

1. Os padrões de melhor prática mudam mais rapidamente do que o tempo que as autoridades regulatórias costumam levar para aprovar novos usos ou emitir novos guias ${ }^{18,19}$;
2. Em muitos casos as "melhores práticas" estabelecidas por governos ou listas oficiais falham em beneficiar os pacientes, de modo que o recurso aos medicamentos em uso off-label e aos resultados de novas pesquisas sobre medicamentos já existentes se torna mais benéfico na abordagem clínica

3. O uso off-label é comum no tratamento de doenças órfãs e de populações específicas como crianças, nas quais a indústria farmacêutica pouco investe em amplos testes para a fase de aprovação de medicamentos ${ }^{7,20,21}$.

Os opositores da prática de prescrição offlabel argumentam que esta poderia ser utilizada como uma forma de burlar esse processo de aprovação de medicamentos lento e caro. Acrescentam que esse tipo de prescrição pode gerar efeitos perversos sobre o sistema regulador, permitindo que as empresas optem por registrar seus produtos pela via ou indicação que apresente maior facilidade durante os estudos clínicos, mesmo que já se saiba de antemão que o público alvo incluirá outras finalidades não contempladas nesses estudos. E tal fato pode acarretar descontrole ao sistema de saúde, em função de gastos em medicamentos e correlatos não previstos ${ }^{1,2,4,7,20,21}$.

Prescrição off-label tem a ver também com incorporação de tecnologia e protocolos custeados pelo sistema de saúde, que precisa estabelecer critérios claros para acesso e pagamento. Em países como os EUA, o pagamento de medicamentos e correlatos prescritos off-label se encontra previsto nos programas Medicare e Medicaid, e pelas legislaturas de diversos estados norte-americanos, que estabelecem regras próprias para custeio de produtos nesta categoria por seguros de saúde. A racionalidade não passa pelo acesso a medicamentos como um direito social, mas como uma responsabilidade securitária, parte intrínseca da prestação de saúde como um contrato $^{2-4,6,7,20,21}$.

No Brasil o fornecimento de medicamentos à população é de responsabilidade estatal, via Sistema Único de Saúde. Cada Estado tem o poder de gerir o seu sistema de assistência farmacêutica, havendo uma superposição de normas de aceitação em programas governamentais, listas de medicamentos essenciais de acordo com realidades locais, formas de compras etc. $\mathrm{O}$ mesmo vale para os municípios, de acordo com o seu nível de gestão dentro do Sistema de Saúde. O resultado é um cenário onde há uma lista básica federal que serve de referência para as listagens das outras esferas da administração, adaptadas às suas realidades locais, bem como programas 
específicos do governo direcionados a problemas como saúde mental, HIV/Aids, doenças sexualmente transmissíveis etc. ${ }^{22,23}$. As formas de acesso aos medicamentos desses programas variam de acordo com as normas que os regulamentam. O uso off-label tende a não ser aceito, mas sim combatido nesses casos.

Por outro lado, associações médicas mostram uma tendência de aceitar o uso off-label como uma prática necessária, incorporada em protocolos e diretrizes, representando consenso de especialistas de diversas áreas sobre procedimentos terapêuticos recomendáveis para condições determinadas, não esgotando as possibilidades de tratamento àquelas descritas, mas sim sugerindo-as como as melhor estabelecidas. A literatura que apóia a rejeição dos protocolos das associações de especialistas alega a grande permeabilidade da classe médica ao marketing da indústria farmacêutica na elaboração desses protocolos, ainda que não possa comprovar diretamente uma relação entre prescrição individual, marketing direto ao prescritor e vendas ${ }^{6,19-21,24-}$ ${ }^{28}$. Entretanto, o sistema de análise, tanto pelas comissões de inclusão em listas governamentais, quanto em protocolos de sociedades de especialistas, se baseia fortemente em análise de literatura publicada em periódicos com corpo editorial independente e com revisão por pares (peer-reviewed), o que não isenta ambos os lados de introduzir um viés do qual este sistema não está imune. "De 38 ensaios vistos como positivos pelo FDA, apenas um não foi publicado. De 36 vistos como negativos ou questionáveis pelo FDA, 22 (61\%) não foram publicados, e outros 11 (31\%) publicados de tal forma que seus resultados passaram uma mensagem mais positiva do que a encontrada pela revisão do FDA. Sob a perspectiva da literatura publicada, 94\% dos ensaios sobre antidepressivos são positivos. Sob a revisão completa e independente do FDA, apenas 51\% dos ensaios foram positivos. A escolha por parte das empresas de não publicar resultados de ensaios nulos ou negativos introduz um sério e não detectável viés na literatura"1-2,5,29,30.

No Brasil a Lei 9.782/9931, que cria a Anvisa, órgão regulador concedente do certificado de Registro de medicamentos, não confere competência para controlar a prática médica, lócus onde se dá a prescrição de medicamentos, mas sim a segurança sanitária na cadeia produtiva entendida como produção e consumo, na forma da legislação. As restrições que a agência impõe sobre a prescrição se limitam ao tipo de receituário que o profissional deverá utilizar, de forma a permi- tir o controle de substâncias comercializadas no mercado e que se encontram sob regime de vigilância sanitária ${ }^{31-33}$.

Como órgão regulador responsável pelo registro de medicamentos e outros produtos de interesse da saúde, na forma da lei, cabe à Anvisa estabelecer normas específicas para aprovação dos mesmos e as regras de controle administrativo fiscal. Como não tem poder sobre a prescrição médica, também não o tem para estabelecer critérios específicos de controle sobre a prática clínica em si. Entretanto, a legalidade da prescrição off-label e a incompetência para controle direto sobre esse tipo de prescrição não retira da autoridade sanitária meios de intervenção.

No Brasil, o controle pode ser feito no processo de registro de produtos, na vigilância póscomercialização, na anuência para concessão de patentes, e no controle da propaganda direcionada principalmente aos prescritores, elos da cadeia produção-consumo onde a vigilância sanitária atua legalmente. Como comparação, vemos a experiência norte-americana que delega a responsabilidade pela concessão de patentes a um órgão específico, sem a consultoria da autoridade sanitária ${ }^{12,14}$.

O controle da propaganda de medicamentos no Brasil é uma obra em andamento cujo ato mais recente é a resolução da diretoria colegiada (RDC) da Anvisa 96/2008 ${ }^{34}$, que “Dispõe sobre a propaganda, publicidade, informação e outras práticas cujo objetivo seja a divulgação ou promoção comercial de medicamentos". Este instrumento regula a propaganda aos prescritores, determinando que as referências bibliográficas utilizadas como apoio às afirmações estejam disponíveis para consulta em Serviços de Atendimento ao Cliente dos produtores, e que estas informações sejam originadas de publicações científicas, com nível de evidência I e II. Quanto à promoção formal em peças publicitárias dos usos e indicações off-label, estes não são permitidos, uma vez que a propaganda deve se ater às informações aprovadas pela Anvisa. Entretanto, a brecha para divulgação se abre na possibilidade de distribuição de artigos científicos, livros técnicos publicados, revistas científicas e outras publicações utilizadas para atualização profissional, conforme a Instrução Normativa no 5 de 20 de Maio de $2009^{35}$. A promoção de produtos para indicações off-label, feita criteriosamente, se caracteriza como disseminação de informação e não é vedada pela legislação brasileira atual.

O Food and Drug Modernization Act (FDAMA), de 1997, que estabeleceu a extensão de seis 
meses de exclusividade patentária a produtos em prescrição pediátrica nos EUA, também incluiu provisões com respeito à propaganda de medicamentos e produtos de saúde off-label. Recentemente, o FDA expôs à consulta novo guia sobre promoção de medicamentos off-label, o qual ainda solicita que material que verse sobre usos offlabel deva ser encaminhado à agência para consolidar pedido de inclusão de indicação a ser formalizado posteriormente $e^{8,12,25,26,36,37}$.

Em 1998, a justiça norte-americana determinou que o FDA passaria a não ter o poder de proibir, restringir, sancionar ou de qualquer maneira limitar a distribuição e a disseminação, por parte de fabricantes de produtos farmacêuticos ou correlatos, de artigos científicos publicados em periódicos profissionais confiáveis com revisão editorial por pares (peer-reviewed), mesmo que esses artigos contenham foco exclusivo ou significativo em usos não aprovados pelo FDA, ou que esses artigos não relatem os artigos originais que basearam a aprovação dos produtos pelo FDA. A justiça determinou que a agência não tem o poder de obstar a disseminação de livros-textos ou de referência ou qualquer parte destes, desde que publicados por editora independente ou de livre comercialização em livrarias ou por outros canais de vendas, mesmo que esses artigos contenham foco exclusivo ou significativo em uso off-label; a agência não pode ainda impedir que o agente regulado sugira palestrantes ou conteúdo para um provedor independente de programação em conjunto com um programa de seminários de educação continuada, ainda que tenham sido inclusos como objeto de discussão usos off-label. O tribunal ressalvou, porém, que se um fabricante prover apoio financeiro para a promoção de qualquer dos materiais supracitados, ele deve: a) revelar seu interesse no produto, b) o fato de que o uso não foi aprovado pelo FDA, c) que seu objetivo é promoção comercial $^{12,26,36-37}$.

\section{Discussão}

Nos Estados Unidos da América (EUA), assim como no Brasil, ocorre a peculiaridade de que se uma droga é aprovada para alguma finalidade, não é ilegal a prescrição para outra diferente, fora de protocolos experimentais específicos ${ }^{1}$.

Opositores da liberdade de prescrever off-label, e que são a favor do teste de todas as indicações e associações antes da liberação para o mercado, parecem avaliar de forma pouco realista as implicações de uma política de testar cada indicação e cada combinação de drogas. Tabarrok ${ }^{5}$ afirma que "Ex post, é fácil apontar combinações perigosas, mas fazer isso indica nada sobre a desejabilidade de regulação ex ante. Como a combinação de drogas é muito comum, o FDA não poderia testar todas as combinações em uso sem um aumento maciço de pessoal e sem reduzir significativamente a adoção de drogas e sem aumentar os custos de inovação. Porque a maioria das combinações são seguras e eficientes, o aumento dos testes de combinações, provavelmente reduziria, mais do que melhoraria, a saúde dos pacientes"1. Testes exaustivos ou excessivos podem trazer complicações regulatórias tais como: aumento do tempo de aprovação de produtos para comercialização, custos imensos de desenvolvimento de produtos pois existem milhares de substâncias e novas são descobertas todos os dias - estes fatores combinados contribuiriam para a demora da chegada de importantes produtos ao consumidor, que não seria beneficiado de maneira alguma ${ }^{1,5}$.

Entretanto, há de se desenvolver políticas eficazes que estimulem a indústria a produzir estudos e drogas para populações órfãs e para pediatria. O caso norte-americano optou por um incentivo patentário, isto é, concedendo extensões de patentes para uma segunda indicação registrada, e um adicional caso o medicamento seja pesquisado em crianças e adolescentes, ainda que os estudos não garantam a aprovação para uso neste público. A lei de drogas órfãs norte americana também permite maiores períodos de tempo de proteção patentária para as drogas desenvolvidas para doenças que atinjam menos de 200.000 pessoas da população americana. Além do incentivo patentário, há incentivos fiscais para as pesquisas e regulatórios como prioridade de análise. As críticas aos incentivos concedidos pela legislação a partir de 1984, com o objetivo incentivar o mercado de genéricos e ao mesmo tempo estimular a pesquisa e a inovação, é a possibilidade que ela abre para sucessivas extensões na duração das patentes e bloqueios processuais ao protocolo de novos genéricos ao final da vigência da patente de um produto. Criou-se na verdade patentes ainda mais longas, de acordo com alguns críticos mais contundentes ${ }^{12,14}$. No Brasil não se observam incentivos formais de natureza patentária, mas sim prioridade na revisão da petição de registro de drogas órfãs.

De resto, a legislação brasileira vigente quanto à utilização off-label ainda carece de maior observação. Neste caso, se abrem campos de pesquisa 
importantes em regulação sanitária e economia ainda não explorados, combinando políticas públicas em saúde, economia, direito e regulação.

\section{Conclusão}

De acordo com o observado neste artigo, o fato da vigilância sanitária no Brasil não possuir competência legal para intervir diretamente na prática de prescrição off-label de medicamentos e produtos de interesse para a saúde não a impede de usar estratégias para exercer algum tipo de controle sobre esta matéria. Como demonstrado acima, as formas de controle utilizadas no Brasil pela Anvisa, a partir de 1999, assemelham-se em vários aspectos às utilizadas pelo FDA nos EUA. As semelhanças residem na necessidade de se confiar nos estudos pós-comercialização, na estruturação de meios de controle sobre a promoção de medicamentos, inclusive para os prescritores, e na necessidade contínua de aperfeiçoar os métodos para aprovação de produtos para comercialização.

Até o presente momento, as indicações de uso se caracterizam como um desfecho a partir do qual se avalia a segurança de produtos para a saúde, e a não inocuidade do produto, propiciando a liberação para consumo, mas não limitando ao consumo para a indicação inicial usada como parâmetro no registro. Isto indica que as limitações do processo de registro, tais como impossibilidade de testar adequadamente todas as indicações possíveis de determinado produto antes do registro inicial e de assumir os riscos de não tratamento, tornam a prescrição off-label uma derivação lógica do atual modelo de vigilância sanitária de produtos e mesmo necessária ${ }^{2,430}$.

Por outro lado, estas questões que são comuns aos sistemas brasileiro e estadunidense, os quais se baseiam numa autoridade centralizada para elaborar e implementar políticas de segurança em saúde, tendem a ser abordadas de maneira diferente, de acordo com valores socialmente construídos, tais como direito à saúde, direitos individuais, liberdade de expressão e informação, limites à autoridade regulatória e intervenção do Estado $^{9,21}$.

Nos EUA, o FDA procura intervir temperado por um ambiente judicial que preserva valores de direitos individuais centrados na liberdade de expressão, na autonomia do indivíduo diante do Estado para formar sua opinião e decidir sobre si mesmo, não constituindo a saúde um direito social conceituado como tal e assim reconhecido $^{11,38}$. No Brasil, a questão saúde envolve outra dinâmica entre o que é público e privado, redesenhando os limites da intervenção da autoridade sobre o que pertence ao indivíduo. Isto se materializa também nas formas escolhidas pela autoridade sanitária de controlar as prescrições médicas que são um momento de interação entre três pessoas: o Estado na forma de regulador da segurança de produtos, o médico como profissional responsável pelos conhecimentos necessários ao tratamento do indivíduo, e o paciente.

Este artigo aponta para a necessidade das pesquisas referentes à utilização de produtos offlabel considerarem os aspectos pertinentes a essa relação triádica, procurando preservar as peculiaridades de cada ponto de vista, sejam eles de formalidade legal, manutenção da segurança das relações jurídicas que regulam o mercado de produtos de interesse da saúde, as necessidades de segurança e eficácia de tratamento que pacientes esperam dos produtos ofertados, a relação entre médico e paciente e a atuação da autoridade reguladora em vigilância sanitária de produtos e serviços no sentido da racionalização e aperfeiçoamento de seus procedimentos de controle. 


\section{Referências}

1. Tabarrok AT. Assessing the FDA Via the Anomaly of Off-Label Prescribing. Independent Review 2000; $\mathrm{V}(5): 25-53$.

2. Beck JM, Azari ED. FDA, off-label use, and informed consent: debunking myths and misconceptions. Food Drug Law J 1998; 53(1):71-104.

3. Boos J. Off label use-label off use? Ann Oncol 2003; 14(1):1-5.

4. Bennett WM. Off-label use of approved drugs: therapeutic opportunity and challenges. $\mathrm{J} \mathrm{Am} \mathrm{Soc} \mathrm{Ne}$ phrol 2004; 15(3):830-831.

5. Tabarrok A. From off-label prescribing towards a new FDA. Med Hypotheses 2009; 72(1):11-13.

6. Gillick MR. Controlling off-label medication use Ann Intern Med 2009; 150(5):344-347.

7. Gazarian M, Kelly M, McPhee JR, Graudins LV, Ward RL, Campbell TJ. Off-label use of medicines: consensus recommendations for evaluating appropriateness. Med J Aust 2006; 185(10):544-548.

8. Azar AM. What a Food and Drug lawyer should know about the Medicare Modernization Act. Food Drug Law J 2004; 59(2):217-222.

9. Piovesan MF. A construção política da Agência Nacional de Vigilância Sanitária [dissertação]. Rio de Janeiro: Fiocruz; 2005.

10. Piovesan MF, Labra ME. Institutional change and political decision-making in the creation of the Brazilian National Health Surveillance Agency. Cad Saude Publica 2007; 23(6):1373-1382.

11. Costa EAd. Vigilância Sanitária - Proteção e Defesa da Saúde. São Paulo: Sociedade Brasileira de Vigilância de Medicamentos; 2004.

12. Parker FR. FDA administrative enforcement manual. Boca Raton: Taylor \& Francis; 2005.

13. Downing GJ. Biomarkers and surrogate endpoints: clinical research and applications: proceedings of the NIH-FDA conference held on 15-16 April 1999 in Bethesda, Maryland, USA: Elsevier Health Sciences; 2000 .

14. Voet MA. The generic challenge : understanding patents, FDA \& pharmaceutical life-cycle management. $2^{\text {nd }}$ Edition. Boca Raton: BrownWalker Press; 2008.

15. Breyer SG. Breaking the vicious circle: toward effective risk regulation. Cambridge: Harvard University Press; 1993.

16. Sunstein CR. The cost-benefit state: the future of regulatory protection. Chicago, Ill.: Section of Administrative Law and Regulatory Practice, ABA; 2002.

17. Berndt ER, Gottschalk AHE, Philpson T, Strobeck MWF. Assessing the impacts of the prescription drug user fee acts (PDUFA) on the FDA approval process. Cambridge: National Bureau of Economic Research; 2004. (Working Paper 10822).

18. Baldwin DS, Kosky N. Off-label prescribing in psychiatric practice. Advances in Psychiatric Treatment 2007; 13:414-422.

19. Lowe-Ponsford FL, Baldwin DS. Off-label prescribing by psychiatrists. Psychiatric Bulletin 2000; 24:415-417.
20. Radley DC, Finkelstein SN, Stafford RS. Off-label prescribing among office-based physicians. Arch Intern Med 2006; 166(9):1021-1026.

21. Stafford RS. Regulating off-label drug use - rethinking the role of the FDA. N Engl J Med 2008; 358(14):1427-1429.

22. Brasil. Ministério da Saúde (MS). Norma Operacional Básica do Sistema Único de Saúde/NOB SUS 96. Brasilia: MS; 1997.

23. Brasil. Ministério da Saúde (MS). Departamento de Atenção Básica. Política Nacional de Medicamentos 2001. Brasília: MS; 2001.

24. Donohue JM, Cevasco M, Rosenthal MB. A decade of direct-to-consumer advertising of prescription drugs. N Engl J Med 2007; 357(7):673-681.

25. Rosenthal MB, Berndt ER, Donohue JM, Frank RG, Epstein AM. Promotion of prescription drugs to consumers. N Engl J Med 2002; 346(7):498-505.

26. Robinson AR, Hohmann KB, Rifkin JI, Topp D, Gilroy CM, Pickard JA, Anderson RJ. Direct-toconsumer pharmaceutical advertising: physician and public opinion and potential effects on the physician-patient relationship. Arch Intern Med 2004; 164(4):427-432.

27. Robinson AR, Hohmann KB, Rifkin JI, Topp D, Gilroy CM, Pickard JA, Anderson RJ. Physician and public opinions on quality of health care and the problem of medical errors. Arch Intern Med 2002; 162(19):2186-2190.

28. Chouinard G. The search for new off-label indications for antidepressant, antianxiety, antipsychotic and anticonvulsant drugs. J Psychiatry Neurosci May 2006; 31(3):168-176.

29. Psaty BM, Ray W. FDA guidance on off-label promotion and the state of the literature from sponsors. JAMA 2008; 299(16):1949-1951.

30. Klein DB, Tabarrok AT. Do Off-Label Drug Practices Argue Against Fda Efficacy Requirements? Testing an Argument by Structured Conversations with Experts. Stockholm: S-WoPEc; 2003.

31. Brasil. Lei 9.782 de 26 de Janeiro de 1999. Define o Sistema Nacional de Vigilância Sanitária, cria a Agência Nacional de Vigilância Sanitária e dá outras providências. Diário Oficial da União 1999; jan 27.

32. Brasil. Lei No 6.360 de 23 de setembro de 1976. Dispõe sobre a vigilância sanitária a que ficam sujeitos os medicamentos, as drogas, os insumos farmacêuticos e correlatos, cosméticos, saneantes e outros produtos, e dá outras providências. Diário Oficial da União 1976; set 24.

33. Brasil. Lei No 5.991 de 17 de dezembro de 1973. Dispõe sobre o Controle Sanitário do Comércio de Drogas, Medicamentos, Insumos Farmacêuticos e Correlatos, e dá outras Providências. Diário Oficial da União 1973; dez 19.

34. Agência Nacional de Vigilância Sanitária (Anvisa). Resolução-RDC no 96 de 17 de dezembro de 2008. Dispõe sobre a propaganda, publicidade e outras práticas cujo objetivo seja a divulgação ou promocão comercial de medicamentos. Brasília: Anvisa; 2008. 
35. Agência Nacional de Vigilância Sanitária (Anvisa). Instrução Normativa no 5 de 20 de maio de 2009. Brasília: Anvisa; 2009.

36. Weeks EA. Is it worth the trouble? The new policy on dissemination of information on off-label drug use under the Food and Drug Administration Modernization Act of 1997. Food Drug Law J 1999; 54(4):645-665.

37. Blum RS. Legal considerations in off-label medication prescribing. Arch Intern Med 2002; 162(15):17771779.

38. Gostin LO. Public health law: power, duty, restraint. $2^{\text {nd }}$ Edition. Berkeley: University of California Press; 2008.

Artigo apresentado em 05/08/2011

Aprovado em 10/09/2011

Versão final apresentada em 05/10/2011 